\title{
SPOLM2019
}

XIX SIMPÓSIO DE PESQUISA OPERACIONAL E LOGÍSTICA DA MARINHA

\section{A PROGRAMAÇÃO MATEMÁTICA COMO FERRAMENTA DE APOIO À DECISÃO PARA UM MICROEMPREENDEDOR INDIVIDUAL (MEI)}

\author{
Kaique de Oliveira Dutton da Silva \\ Centro de Tecnologia da indústria Química e Têxtil (SENAI CETIQT) \\ Rua Magalhães Castro 174 - Riachuelo - Rio de Janeiro - RJ - Brasil \\ kaiquedutton@gmail.com \\ Marcos dos Santos \\ Instituto Militar de Engenharia (IME) \\ Praça General Tibúrcio, 80 - Praia Vermelha - Urca - Rio de Janeiro - RJ \\ marcosdossantos_doutorado_uff@yahoo.com.br \\ Marcone Freitas dos Reis \\ Centro de Tecnologia da indústria Química e Têxtil (SENAI CETIQT) \\ Rua Magalhães Castro 174 - Riachuelo - Rio de Janeiro - RJ - Brasil \\ marconefreis11@gmail.com \\ Ernesto Rademaker Martins \\ Centro de Análise de Sistemas Navais (CASNAV) \\ Praça Barão de Ladário s/n - Ilha das Cobras - Rio de Janeiro - RJ - CEP: 20091-000 \\ radmart@yahoo.com.br
}

\section{RESUMO}

Com o passar dos anos, o comércio varejista vem sofrendo inúmeras mudanças e o segmento que estiver buscando a melhora contínua da qualidade de seus serviços certamente terá destaque neste tipo de ramo. A maximização do lucro é uma tarefa complicada, pois eventualmente o comerciante que fazer esta ação sem investir muito e para a obtenção de tal feito podem-se citar as técnicas da Pesquisa Operacional, mas certamente os modelos determinísticos, no qual a programação matemática está inserida. O objetivo desta pesquisa é propor um mix de produtos visando a maximização do lucro nominal de um microempreendedor individual. A metodologia utilizada para a tentativa de tal feito foi a Programação Linear Inteira (PLI). Com a utilização desta ferramenta, foi possível obter um lucro nominal de $\mathrm{R}$ \$ 2.916,68 em apenas um dia de trabalho, visto que a função objetivo alcançou um valor de $\mathrm{R} \$$ 8.916,479 e os produtos a serem comercializados tiveram um custo de $\mathrm{R} \$ 5.999,80$. O resultado da pesquisa alcançou o seu êxito mediante os cenários propostos.

Palavra-chave: Comércio Varejista; Pesquisa Operacional; Mix de Produtos; Programação Linear Inteira (PLI) 


\begin{abstract}
Over the years, the retail trade has undergone numerous changes and the segment that is seeking the continuous improvement of the quality of its services will certainly be highlighted in this type of branch. Profit maximization is a complicated task, because eventually the trader who does this action without investing too much and in order to obtain such a feat can cite the techniques of Operational Research, but certainly the deterministic models in which mathematical programming is embedded. . The objective of this research is to propose a product mix aimed at maximizing the nominal profit of an individual microentrepreneur. The methodology used to attempt this was the Integer Linear Programming (PLI). Using this tool, it was possible to obtain a nominal profit of $\mathrm{R} \$$ $2,916.68$ in just one working day, since the objective function reached a value of $\mathrm{R} \$$ $8,916,479$ and the products to be traded had a cost of $\mathrm{R} \$ 5,999.80$. The research result achieved its success through the proposed scenarios.
\end{abstract}

Keywords: Retail Business; Operational Research; Product Mix; Integer Linear Programming

\title{
Como Citar:
}

SILVA, Kaique de Oliveira Dutton da; SANTOS, Marcos dos Santos; REIS, Marcone Freitas dos; MARTINS, Ernesto Rademaker. A Programação Matemática como ferramenta de apoio à decisão para um microempreendedor individual (MEI). In: SIMPÓSIO DE PESQUISA OPERACIONAL E LOGÍSTICA DA MARINHA, 19., 2019, Rio de Janeiro, RJ. Anais [...]. Rio de Janeiro: Centro de Análises de Sistemas Navais, 2019.

\section{INTRODUÇÃO}

Uma feira livre pode ser caracterizada como um espaço ao ar livre onde os comerciantes montam suas barracas e acondicionam seus produtos, sejam eles de qualquer natureza, e os comercializa. Este tipo de ramo mostrou-se muito resistente com o passar dos nãos, mesmo com as recentes inovações contemporâneas dos mercados, que abastecem a população como um todo. Este segmento é uma modalidade muito antiga no mundo, tendo relatos de que foi criada na Idade Média por volta do século IX. O segmento da feira emprega diversas pessoas no país e gera uma movimentação considerável no mercado de produtos alimentícios.

Para Silva et al (2014) as feiras livres são um complexo de relações sociais e econômicas que ocorrem dentro de um determinado espaço público e apresentam relevância inegável, principalmente no nordeste brasileiro, por serem, muitas vezes, a única fonte de renda de inúmeras famílias que não encontraram outra atividade econômica alternativa de sobrevivência. 
desenvolvimento local, pois garante a comercialização da produção familiar, da pequena agroindústria e de produtos artesanais. A feira também favorece outros setores da economia, através da circulação pelos feirantes, que após a comercialização de seus produtos, costumam comprar à vista em vários estabelecimentos do município, tais como supermercados, lojas de roupa, sapatos, produtos agrícolas, farmácias, material de construção entre outros (ARAUJO et al., 2011 p.5).

As feiras são fenômenos econômicos, sociais e culturais muito antigos e no Brasil estão presentes desde o Período Colonial e além disso, no interior do Nordeste elas ainda não desapareceram, mesmo sofrendo com as consequências das modernizações do atual período técnico-científico e informacional (CHAVES, 2011).

Segundo Cunha (2014), além do desempenho no fornecimento de alimentos, a feira livre também se destaca na geração de empregos, visto não é necessária a mão de obra muito qualificada para executar o trabalho. Ainda pode-se considerar outros tipos de serviços realizados na feira como geradores de emprego, sendo: frete, transporte, vendedores, ambulantes, vendedores de lanches etc.

\section{FUNDAMENTAÇÃO TEÓRICA}

\subsection{Comércio Varejista}

Segundo Kotler (2006), o varejo inclui todas as atividades relativas à venda de produtos ou serviços diretamente ao consumidor final, para uso pessoal ou não, e que, além disso, um varejista ou uma loja de varejo é qualquer empreendimento comercial cujo faturamento provenha principalmente da venda de pequenos lotes de varejo.

O varejo é um local onde se realiza trocas de valor, trocas essas objetivas e subjetivas, onde por um lado o cliente recebe valores como produtos físicos, serviços, conveniência, experiências e por vezes entretenimento, e por outro paga valores monetários pela prestação desse serviço (CHAMIE et al., 2012).

Guerra et al. (2013), consideram que o varejo proporciona maior qualidade de vida, através da oferta de produtos que saciem a necessidade dos clientes, gerando renda para movimentar a economia local podendo ser classificado como varejo com loja e varejo sem loja. Segundo Kotler e Armstrong (2007), o varejo sem lojas vem crescendo consideravelmente, abrangendo vendas por mala-direta, catálogos, telefone, internet, televisão, reuniões, contato de porta em porta, dentre outras maneiras.

Nos últimos anos, o comércio varejista vem se destacando na economia brasileira empregando um número maior de pessoas do que o setor industrial e se revelando propulsor 
econômico de diversos outros países é definido usualmente, como elo entre fornecedores e consumidor final (BRITO et al., 2017). O setor varejista, além de responsável por parte considerável da geração de renda nacional, é um termômetro importante da economia, refletindo tendências diversas, como o comportamento do consumo das famílias e a evolução de vendas industriais no mercado interno (ALMEIDA et al., 2016).

\subsection{Pesquisa Operacional}

A Pesquisa Operacional ou ainda PO surgiu na Inglaterra durante a Segunda Guerra Mundial para a solução de problemas de natureza logística, tática e estratégia militar, quando um grupo de cientistas foi convocado para decidir sobre como utilizar de maneira mais eficaz os recursos militares limitados (BELFIORE e FÁVERO, 2013). Conforme Briesemeister e Borba (2014), a PO é um ramo da matemática aplicada destinada a resolver problemas de decisão e faz uso de modelos matemáticos, estatísticos e de algoritmos que auxiliam na resolução de problemas complexos do mundo real, tipicamente com o objetivo de melhorar ou otimizar o desempenho.

Segundo Moreira (2013), a Pesquisa Operacional lida com problemas de como conduzir e coordenar operações em uma organização, e tem sido aplicada a diversas áreas, tais como: indústria, finanças, saúde, serviços públicos, operações militares, entre outros. Segundo Arenales et al. (2015), a abordagem de resolução de um problema por meio de pesquisa operacional envolve várias fases, tais como: definição do problema, construção do modelo, solução do modelo, validação do modelo e implementação da solução.

\subsection{Programação Linear Inteira}

De acordo com Belfiore e Fávero (2013), um problema é classificado como sendo de programação inteira (PI) quando todas as variáveis de decisão do modelo são discretas, isto é, podem assumir valores dentro de um conjunto finito ou uma quantidade enumerável de valores, provenientes de uma contagem. Para Lachtermacher (2007), programação inteira envolve situações de programação matemática em que todas as funções envolvidas são lineares, mas uma das condições é que o valor das variáveis discriminadas sejam valores inteiros.

Além da necessidade de restringir as variáveis a serem inteiras, outra diferença em problema de programação inteira é que estes mesmos problemas têm um número mais reduzido de soluções a serem consideradas e limitadas, com um número finito de soluções 
viáveis (PEIXOTO et al., 2017). Um dos algoritmos mais utilizados para a resolução de problemas é o algoritmo de Branch and Bound (B\&B) (TEIXEIRA et al., 2017). A Figura 2 a seguir mostra o diagrama de funcionamento do algoritmo Branch and Bound.

Figura 1: Diagrama de funcionamento do algoritmo Branch and Bound

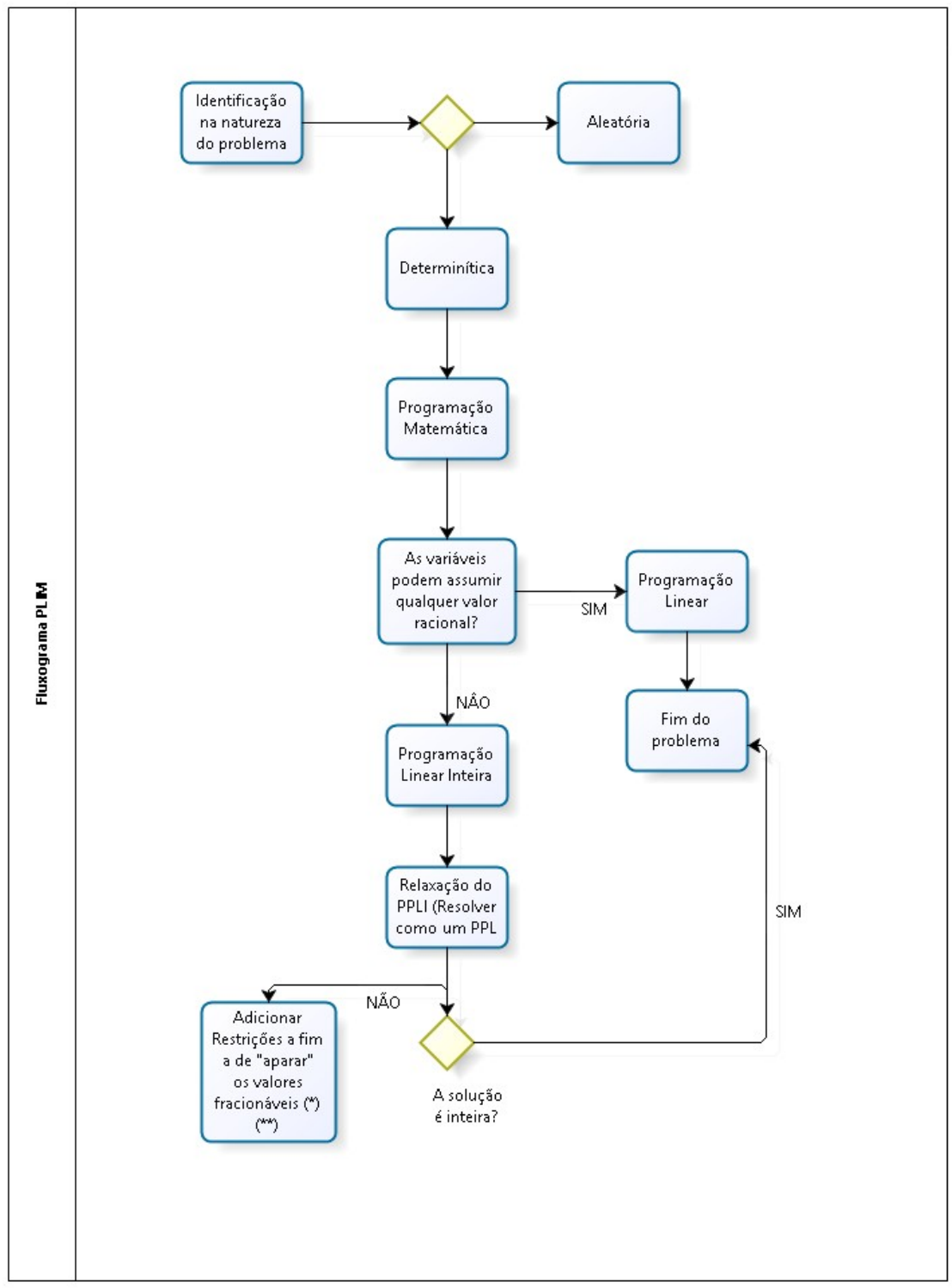

Fonte: Autores (2019)

\section{DESCRIÇÂO DO PROBLEMA}

Assim como nas empresas, as barracas de uma feira livre têm de se adaptar às mudanças econômicas para prestar um atendimento melhor ao seu cliente. Na feira existe 
muita concorrência entre os trabalhadores e os mesmos recorrem a frases de efeito para tentar atrair o olhar do cliente e posteriormente sua confiança. Os feirantes são trabalhadores que acordam ainda pela madrugada para ir até o CEASA-RJ e adquirir seus produtos para serem vendidos.

O problema em questão é determinar o mix de produtos que maximizem o lucro nominal de um proprietário de uma barraca, pertencente a uma feira livre no município do Rio de Janeiro, respeitando-se as restrições de fluxo de caixa e de capacidade do volume transportado.

Para a obtenção dos produtos a serem vendidos pelo comerciante, ele dispõe de R $\$$ 6.000,00. Pode-se dizer que parte do problema é salientar quanto o trabalhador gasta na aquisição de seus produtos, estipular a demanda diária dos produtos com maior relevância de retorno capital, fazer a medição destes produtos selecionados e dos locais onde estas mercadorias são acondicionadas a fim de se respeitar a capacidade do volume transportado e estimar esta demanda aplicando-se a ela 20 pontos percentuais para mais ou menos a fim de se obter as restrições de demanda que o comerciante possui. Com a posse desses dados podese montar as equações e restrições que fazem parte do problema.

Para a montagem da função objetivo, foi utilizado o preço de venda de cada produto, ou seja, já padronizado como sendo o preço da embalagem da mercadoria, preço do quilo multiplicado pelo peso da embalagem. Assim define-se a função objetivo de maximização do problema. Agora, pode-se definir as restrições de capacidade do volume transportado. Para a obtenção da restrição chamada de "Geladeira", foram utilizados os valores correspondentes ao volume ocupado por cada produto na geladeira com sua respectiva variável de decisão. A equação da restrição denominada "Kombi” foi obtida através dos valores correspondentes ao volume ocupado por cada item que compõe a Kombi (parte de trás) multiplicada por sua respectiva variável de decisão. A equação de nominada "Fluxo de Caixa" foi obtida através do preço de aquisição de cada item com sua respectiva variável de decisão.

Após a obtenção das equações que compõem o problema, foi feita sua modelagem com o auxílio do software Linear Interative and Discrete Optimize (LINDO). Na tentativa de obtenção da solução ótima, foram propostos pelo autor dois cenários do problema. $\mathrm{O}$ primeiro cenário consta a modelagem do problema sem as restrições referentes à demanda diária e o segundo cenário de solução entram as demandas diárias de cada item. 
Para a melhor compreensão e delimitação das condições de contorno do problema, foi construído pelo autor um mapa mental, o qual consta na Figura 2 a seguir.

Figura 2: Mapa Mental

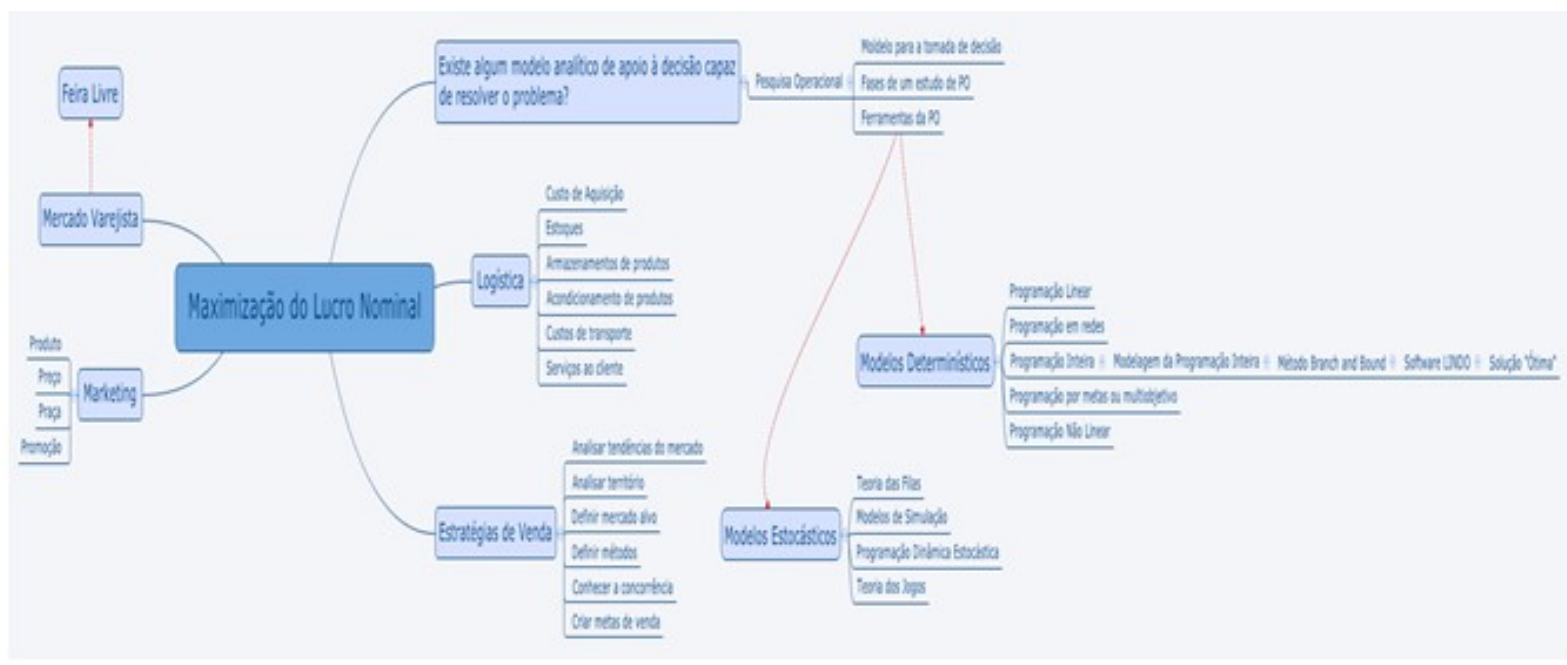

Fonte: Autores (2019)

\section{MODELAGEM MATEMÁTICA}

A Tabela 1 a seguir mostra os produtos que são vendidos pelo comerciante e suas respectivas variáveis de decisão, além de mostrar o volume dos itens, os preços de aquisição e venda e o lucro obtido. Vale ressaltar que o motivo pelo qual foram feitas as medições e as pesagens dos produtos foi para se obter os preços das embalagens das mercadorias para facilitar a montagem da função objetivo e das restrições.

Tabela 1: Visão Geral dos dados do problema

\begin{tabular}{ccccccc}
\hline Item & $\begin{array}{c}\text { Unidade de } \\
\text { fornecimento }\end{array}$ & Volume & $\begin{array}{c}\text { Variável } \\
\text { de } \\
\text { decisão }\end{array}$ & $\begin{array}{c}\text { Venda } \\
\text { (R\$) }\end{array}$ & $\begin{array}{c}\text { Compra } \\
\text { (R\$) }\end{array}$ & Lucro \\
\hline $\begin{array}{c}\text { Ovo de } \\
\text { codorna } \\
\text { Ovo }\end{array}$ & Dúzia & $560 \mathrm{~cm}^{3}$ & $X_{1}$ & 5,00 & 3,00 & 2,00 \\
$\begin{array}{c}\text { Branco } \\
\text { Ovo }\end{array}$ & Dúzia & $1458 \mathrm{~cm}^{3}$ & $X_{2}$ & 5,00 & 3,50 & 1,50 \\
$\begin{array}{c}\text { Vermelho } \\
\text { Coxa de }\end{array}$ & Embalagem & $1458 \mathrm{~cm}^{3}$ & $X_{3}$ & 7,00 & 5,00 & 2,00 \\
$\begin{array}{c}\text { Frango } \\
\text { Picanha }\end{array}$ & Embalagem & $630 \mathrm{~cm}^{3}$ & $X_{4}$ & 12,90 & 7,00 & 5,90 \\
$\begin{array}{c}\text { Suína } \\
\text { Picanha }\end{array}$ & Embalagem & $1512 \mathrm{~cm}^{3}$ & $X_{5}$ & 26,90 & 18,80 & 8,10 \\
Bovina & $X_{6}$ & 49,40 & 36,40 & 13,00 \\
$\begin{array}{c}\text { Miúdo de } \\
\text { Porco }\end{array}$ & Embalagem & 13950 & $X_{7}$ & 38,70 & 24,00 & 14,70 \\
$\mathrm{~cm}^{3}$ & & & &
\end{tabular}




\begin{tabular}{|c|c|c|c|c|c|c|}
\hline $\begin{array}{l}\text { Pé de } \\
\text { Frango }\end{array}$ & Caixa & $\begin{array}{c}112404 \\
\mathrm{~cm}^{3}\end{array}$ & $X_{8}$ & 85,00 & 34,00 & 51,00 \\
\hline Carré & Embalagem & $\begin{array}{c}20160 \\
\mathrm{~cm}^{3}\end{array}$ & $X_{9}$ & 81,12 & 48,00 & 33,12 \\
\hline $\begin{array}{l}\text { Carne } \\
\text { Moída }\end{array}$ & Embalagem & $\begin{array}{c}15876 \\
\mathrm{~cm}^{3}\end{array}$ & $X_{10}$ & 48,00 & 40,00 & 8,00 \\
\hline $\begin{array}{l}\text { Costela } \\
\text { de Porco }\end{array}$ & Embalagem & $\begin{array}{c}30618 \\
\mathrm{~cm}^{3}\end{array}$ & $X_{11}$ & 39,60 & 24,00 & 15,60 \\
\hline $\begin{array}{l}\text { Carcaça } \\
\text { de Frango }\end{array}$ & Embalagem & $5985 \mathrm{~cm}^{3}$ & $X_{12}$ & 5,00 & 3,00 & 2,00 \\
\hline $\begin{array}{l}\text { Coração } \\
\text { de Frango }\end{array}$ & Embalagem & $560 \mathrm{~cm}^{3}$ & $X_{13}$ & 19,00 & 16,00 & 3,00 \\
\hline Pescoço & Embalagem & $4851 \mathrm{~cm}^{3}$ & $X_{14}$ & 5,00 & 2,00 & 3,00 \\
\hline $\begin{array}{c}\text { Sobrecox } \\
a\end{array}$ & Embalagem & $7942 \mathrm{~cm}^{3}$ & $X_{15}$ & 25,80 & 14,00 & 11,80 \\
\hline Alcatra & Embalagem & $\begin{array}{c}12064 \\
\mathrm{~cm}^{3}\end{array}$ & $X_{16}$ & 156,87 & 113,40 & 43,47 \\
\hline $\begin{array}{c}\text { Filé } \\
\text { Mignon }\end{array}$ & Embalagem & $\begin{array}{c}33840 \\
\mathrm{~cm}^{3}\end{array}$ & $X_{17}$ & 90,00 & 70,00 & 20,00 \\
\hline $\begin{array}{c}\text { Peito de } \\
\text { Frango }\end{array}$ & Embalagem & $\begin{array}{c}11232 \\
\mathrm{~cm}^{3}\end{array}$ & $X_{18}$ & 12,90 & 8,00 & 4,90 \\
\hline $\begin{array}{l}\text { Linguiça } \\
\text { de Frango }\end{array}$ & Embalagem & $\begin{array}{c}10800 \\
\mathrm{~cm}^{3}\end{array}$ & $X_{19}$ & 7,92 & 5,56 & 2,36 \\
\hline $\begin{array}{l}\text { Carne } \\
\text { Seca }\end{array}$ & Embalagem & $\begin{array}{c}40824 \\
\mathrm{~cm}^{3}\end{array}$ & $X_{20}$ & 289,00 & 200,00 & 89,00 \\
\hline $\begin{array}{c}\text { Carne de } \\
\text { Sol }\end{array}$ & Embalagem & $\begin{array}{c}40824 \\
\mathrm{~cm}^{3}\end{array}$ & $X_{21}$ & 289,00 & 200,00 & 89,00 \\
\hline Toucinho & Embalagem & $7830 \mathrm{~cm}^{3}$ & $X_{22}$ & 13,90 & 10,00 & 3,90 \\
\hline
\end{tabular}

Fonte: Autores (2019)

Com a posse desses dados foram montadas a função objetivo do problema e as restrições que o compõem.

\subsection{Função Objetivo}

A função objetivo do problema consiste em maximizar o lucro nominal do comerciante e, como foi mencionado antes, ela foi construída a partir do valor do preço de compra dos produtos multiplicado pela sua respectiva variável de decisão. A seguir pode-se ver a função objetivo modelada.

F.O $=\max \left\{5 X_{1}+5 X_{2}+7 X_{3}+12.9 X_{4}+26.9 X_{5}+49.4 X_{6}+38.7 X_{7}+85 X_{8}+81.12 X_{9}+48 X_{10}+39.6 X_{11}+5 X_{12}\right.$

\subsection{Restrições}


Neste tópico serão apresentadas as restrições que compõem o modelo matemático proposto.

\subsubsection{Geladeira}

Como foi mencionado em um tópico anterior, a restrição para a geladeira foi obtida com o volume dos produtos que a compõe. A equação para a restrição da geladeira pode ser observada a seguir:

Geladeira:

$640 X_{4}+630 X_{5}+1512 X_{6}+13950 X_{7}+112404 X_{8}+20160 X_{9}+15876 X_{10}+30618 X_{11}+5985 X_{12}+560 X_{13}+48$

\subsubsection{Kombi}

Como já mencionado antes, a restrição para a Kombi foi obtida através dos itens que o compõe. A equação para a restrição da Kombi pode ser vista a seguir.

Fluxo

de caixa:

$3 X_{1}+3.5 X_{2}+5 X_{3}+7 X_{4}+18.8 X_{5}+36.4 X_{6}+24 X_{7}+34 X_{8}+48 X_{9}+40 X_{10}+24 X_{11}+3 X_{12}+16 X_{13}+2 X_{14}+14$

\subsubsection{Não Negatividade}

Considerando $X_{i}$ a quantidade de cada produto a ser vendido, a restrição de não negatividade pode ser vista a seguir:

$X_{i} \geq 0$ para todo $i=\{1,2,3,4,5,6,7,8 \ldots 22\}$

\subsubsection{Integralidade}

$X_{i} \in Z \square / \square i \in\{1,2,3,4,5,6,7,8 \ldots 22\}$

\subsection{Solução do modelo}

O software LINDO foi escolhido para a determinação da solução ótima do problema proposto, ou seja, mostrando a quantidade de cada produto que deve ser vendido para a maximização do lucro do proprietário da barraca. Para atingir tal objetivo, foram modelados no software a função objetivo e as restrições do problema. A Figura 3 mostra a solução do modelo proposto. 
Figura 3: Solução do cenário 1 do problema

\begin{tabular}{ccr}
\multicolumn{3}{c}{ OBJECTIVE FUNCTION VALUE } \\
1) & 11287.40 & \\
VARIABLE & VALUE & REDUCED COST \\
X1 & 2.000000 & -5.000000 \\
X2 & 0.000000 & -5.000000 \\
X3 & 0.000000 & -7.000000 \\
X4 & 806.000000 & -12.900000 \\
X5 & 0.000000 & -26.900000 \\
X6 & 0.000000 & -49.900002 \\
X7 & 0.000000 & -38.700001 \\
X8 & 0.000000 & -85.000000 \\
X9 & 0.000000 & -81.120003 \\
X10 & 0.000000 & -48.000000 \\
X11 & 0.000000 & -39.599998 \\
X12 & 0.000000 & -5.000000 \\
X13 & 0.000000 & -19.000000 \\
X14 & 176.000000 & -5.000000 \\
X15 & 0.000000 & -25.799999 \\
X16 & 0.000000 & -156.869995 \\
X17 & 0.000000 & -90.000000 \\
X18 & 0.000000 & -12.900000 \\
X19 & 0.000000 & -7.920000 \\
X20 & 0.000000 & -289.000000 \\
X21 & 0.000000 & -289.000000 \\
X22 & 0.000000 & -13.900000 \\
& &
\end{tabular}

Fonte: Autores (2019)

Com a solução do cenário 1 do problema, pode ser observado que a solução ainda não é satisfatória, pois quase todas as variáveis de decisão resultaram em valor zero, com exceção das variáveis X1, X4 e X14 que apresentaram valores não nulos. Para a obtenção de uma solução mais satisfatória que a anterior, foi proposto um novo cenário, no qual entram as restrições de demanda diária na tentativa de formar um mix mais realista de produtos a serem ofertados em um dia de trabalho na feira. Vale ressaltar que as demandas diárias de cada produto que compõem o problema foram obtidas por meio de entrevistas com o comerciante. $\mathrm{O}$ item a seguir mostrará o novo cenário.

\subsection{Cenário Proposto}

Como mencionado antes, as restrições de demanda diária entram no novo cenário e a Tabela 2 a seguir mostra a demanda diária dos itens e mostra também os pontos percentuais que foram impostos para a tentativa de obtenção do mix ótimo.

Tabela 2: Demanda diária dos produtos com porcentagem

\begin{tabular}{cccc}
\hline Item & Variável de decisão & Demanda diária & Demanda +/- 20\% \\
\hline Ovo de codorna & $X_{1}$ & $180 \mathrm{Dz}$ & 144 a 216 \\
Ovo branco & $\mathrm{X}_{2}$ & $90 \mathrm{Dz}$ & 72 a 108 \\
Ovo vermelho & $\mathrm{X}_{3}$ & $60 \mathrm{dz}$ & 48 a 72 \\
Coxa de frango & $\mathrm{X}_{4}$ & $24 \mathrm{Kg}$ & 19,2 a 28,8 \\
Picanha suína & $\mathrm{X}_{5}$ & $6 \mathrm{Kg}$ & 4,8 a 7,2 \\
Picanha bovina & $\mathrm{X}_{6}$ & $7 \mathrm{Kg}$ & 5,6 a 8,4 \\
Miúdo de porco & $\mathrm{X}_{7}$ & $7 \mathrm{Kg}$ & 5,6 a 8,4
\end{tabular}




\begin{tabular}{cccc} 
Pé de frango & $\mathrm{X}_{8}$ & $36 \mathrm{Kg}$ & 28,8 a 43,2 \\
Carré & $\mathrm{X}_{9}$ & $10 \mathrm{Kg}$ & 8 a 12 \\
Carne moída & $\mathrm{X}_{10}$ & $6 \mathrm{Kg}$ & 4,8 a 7,2 \\
Costela de porco & $\mathrm{X}_{11}$ & $12 \mathrm{Kg}$ & 9,6 a 14,4 \\
Carcaça de frango & $\mathrm{X}_{12}$ & $4 \mathrm{Kg}$ & 3,2 a 4,8 \\
Coração de frango & $\mathrm{X}_{13}$ & $5 \mathrm{Kg}$ & 4 a 6 \\
Pescoço & $\mathrm{X}_{14}$ & $10 \mathrm{Kg}$ & 8 a 12 \\
Sobrecoxa & $\mathrm{X}_{15}$ & $24 \mathrm{Kg}$ & 19,2 a 28,8 \\
Alcatra & $X_{16}$ & $7 \mathrm{Kg}$ & 5,6 a 8,4 \\
Filé Mignon & $X_{17}$ & $15 \mathrm{Kg}$ & 12 a 18 \\
Peito de frango & $X_{18}$ & $48 \mathrm{Kg}$ & 38,4 a 57,6 \\
Linguiça de frango & $X_{19}$ & $4 \mathrm{Kg}$ & 3,2 a 4,8 \\
Carne Seca & $X_{20}$ & $5 \mathrm{Kg}$ & 4 a 6 \\
Carne se Sol & $X_{21}$ & $5 \mathrm{Kg}$ & 4 a 6 \\
Toucinho & $X_{22}$ & $5 \mathrm{Kg}$ & 4 a 6 \\
\hline
\end{tabular}

Fonte: Autores (2019)

Com a posse desses dados, foi feita novamente a modelagem do novo cenário do problema no software. As restrições de demanda foram feitas respeitando-se os percentuais máximos e mínimos de cada produto. A Figura 4 mostra a solução do novo cenário.

Figura 4: Solução do cenário 2 do problema

OBJECTIVE FUNCTION VALUE

1)

8916.479

VARIABLE
X1
X2
X3
X4
X5
X6
X7
X8
X9
X10
X11
X12
X13
X14
X15
X16
X17
X18
X19
X20
X21
X22

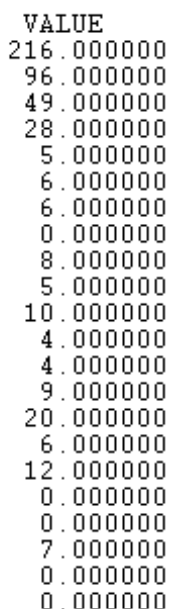

REDUCED COST
-5.000000

$-5.000000$

$-7.000000$

$-12.900000$

$-26.900000$

$-49.900002$

$-38.700001$

$-85.000000$

$-81.120003$

$-48.000000$

$-39.599998$

$-5.000000$

$-19.000000$

$-5.000000$

$-25.799999$

$-156.869995$

$-90.000000$

$-12.900000$

$-7.920000$

$-289.000000$

$-289.000000$

$-13.900000$

Fonte: Autores (2019)

\section{DISCUSSÃO DOS RESULTADOS}

Após a aplicação do software LINDO na solução dos dois cenários propostos, podese observar que a solução do cenário 1 não foi satisfatória, pois quase todos os produtos 
obtiveram valor zero com exceção das variáveis X1, X4 e X14. Então para a obtenção de uma solução mais consistente, foi proposto um novo cenário, no qual entravam as demandas diárias dos produtos com o objetivo de fazer que as variáveis de decisão obtivessem valores inteiros. O comerciante tinha um fluxo de caixa de $\mathrm{R} \$ 6.000,00$, com a solução proposta foi gasto em mercadorias o valor de $\mathrm{R} \$ 5.999,80$ conforme mostra a Tabela 3.

Tabela 3: Custo das mercadorias

\begin{tabular}{|c|c|c|c|c|}
\hline Item & $\begin{array}{l}\text { Variável de } \\
\text { decisão }\end{array}$ & $\begin{array}{l}\text { Valor da } \\
\text { variável de } \\
\text { decisão } \\
\text { (software) }\end{array}$ & $\begin{array}{c}\text { Preço de } \\
\text { aquisição de } \\
\text { cada produto }\end{array}$ & $\begin{array}{c}\text { Total (Variável } \\
\text { de decisão X } \\
\text { preço de } \\
\text { aquisição) }\end{array}$ \\
\hline $\begin{array}{l}\text { Ovo de } \\
\text { codorna }\end{array}$ & $X_{1}$ & 216.000 & $R \$ 3,00$ & $\mathrm{R} \$ 648,00$ \\
\hline Ovo branco & $x_{2}$ & 96.000 & $R \$ 3,50$ & $\mathrm{R} \$ 336,00$ \\
\hline Ovo vermelho & $X_{3}$ & 49.000 & $\mathrm{R} \$ 5,00$ & $\mathrm{R} \$ 245,00$ \\
\hline Coxa de frango & $X_{4}$ & 28.000 & $\mathrm{R} \$ 7,00$ & $\mathrm{R} \$ 196,00$ \\
\hline Picanha suína & $X_{5}$ & 5.000 & $R \$ 18,80$ & $R \$ 94,00$ \\
\hline Picanha bovina & $X_{6}$ & 6.000 & $R \$ 36,40$ & $\mathrm{R} \$ 218,40$ \\
\hline $\begin{array}{l}\text { Miúdo de } \\
\text { porco }\end{array}$ & $X_{7}$ & 6.000 & $R \$ 24,00$ & $\mathrm{R} \$ 144,00$ \\
\hline Pé de frango & $X_{8}$ & 0.000 & $\mathrm{R} \$ 34,00$ & $\mathrm{R} \$ \mathbf{0 , 0 0}$ \\
\hline Carré & $X_{9}$ & 8.000 & $R \$ 48,00$ & $\mathrm{R} \$ 384,00$ \\
\hline Carne moída & $x_{10}$ & 5.000 & $R \$ 40,00$ & $R \$ 200,00$ \\
\hline $\begin{array}{c}\text { Costela de } \\
\text { porco }\end{array}$ & $X_{11}$ & 10.000 & $R \$ 24,00$ & $\mathrm{R} \$ 240,00$ \\
\hline $\begin{array}{c}\text { Carcaça de } \\
\text { frango }\end{array}$ & $X_{12}$ & 4.000 & $R \$ 3,00$ & $\mathrm{R} \$ 12,00$ \\
\hline $\begin{array}{l}\text { Coração de } \\
\text { frango }\end{array}$ & $X_{13}$ & 4.000 & $R \$ 16,00$ & $\mathrm{R} \$ 64,00$ \\
\hline Pescoço & $X_{14}$ & 9.000 & $\mathrm{R} \$ 2,00$ & $\mathrm{R} \$ 18,00$ \\
\hline Sobrecoxa & $\mathrm{X}_{15}$ & 20.000 & $R \$ 14,00$ & $\mathrm{R} \$ 280,00$ \\
\hline Alcatra & $X_{16}$ & 6.000 & $R \$ 113,40$ & $\mathrm{R} \$ 680,40$ \\
\hline Filé Mignon & $X_{17}$ & 12.000 & $\mathrm{R} \$ 70,00$ & $\mathrm{R} \$ 840,00$ \\
\hline $\begin{array}{l}\text { Peito de } \\
\text { frango }\end{array}$ & $\mathrm{X}_{18}$ & 0.000 & $\mathrm{R} \$ 8,00$ & $\mathrm{R} \$ 0,00$ \\
\hline $\begin{array}{l}\text { Linguiça de } \\
\text { frango }\end{array}$ & $\mathrm{X}_{19}$ & 0.000 & $\mathrm{R} \$ 5,56$ & $\mathrm{R} \$ 0,00$ \\
\hline Carne seca & $x_{20}$ & 7.000 & $R \$ 200,00$ & $\mathrm{R} \$ 1.400,00$ \\
\hline Carne de sol & $x_{21}$ & 0.000 & $R \$ 200,00$ & $\mathrm{R} \$ 0,00$ \\
\hline \multirow[t]{2}{*}{ Toucinho } & $X_{22}$ & 0.000 & $\mathrm{R} \$ 10,00$ & $R \$ 0,00$ \\
\hline & & & & $\mathrm{R} \$ 5999,80$ \\
\hline
\end{tabular}


Como mostra a Tabela 3, o total com a compra das mercadorias foi obtido através da multiplicação do valor da variável, gerada no software, com o valor de aquisição dos produtos. Como foi visto, na solução do cenário 2, a função objetivo alcançou um resultado de $\mathrm{R} \$ 8.916,479$ e como foi visto na Tabela 3, o total de compra de mercadorias foi de $\mathrm{R} \$$ 5.999,80. A partir desses dados, pode-se calcular o lucro que o comerciante, ou seja, $\mathrm{R} \$ 8.916,479$ - R $\$$ 5.999,80 obtendo-se um valor de $\mathrm{R} \$ 2.916,68$.

Este valor apresentado representa o lucro do comerciante, o que mostra que este valor é bastante satisfatório e atende ao cenário proposto anteriormente, pois o comerciante tinha um fluxo de caixa de $\mathrm{R} \$ 6.000,00$, gasta $\mathrm{R} \$ 5.999,80$ na aquisição dos produtos e tem um lucro de $\mathrm{R} \$ 2.916,68$ considerando que todas as mercadorias serão vendidas.

\section{CONSIDERAÇÕES FINAIS}

A Programação Linear Inteira (PLI) não é uma novidade no meio acadêmico, contudo, percebe-se uma certa resistência dos proprietários de negócios ou do setor produtivo em usar essas técnicas oriundas da academia, pois estes proprietários pensam que já estão vendendo seus produtos ou serviços da melhor maneira possível. Muitos empresários ou até mesmo gerentes mostram-se muito pessimistas em pensar que existe algum modelo analítico que trará algum resultado prático para seu negócio ou sua empresa.

Comparando-se os dois cenários do presente problema, foi visto que no cenário 1 , onde não entravam as restrições de demanda, foi obtido um resultado bastante insatisfatório, o que não correspondia a realidade do proprietário da barraca, ou seja, foi obtido um valor de R\$ $11.287,40$ na função objetivo e apenas três produtos obtiveram valores não nulos não resolvendo o problema em tela. Na tentativa de se obter um mix mais realista, foi proposto o cenário 2, no qual entravam as demandas diárias e foi visto que neste cenário, obteve-se um valor mais satisfatório que o valor anterior, pois o valor da função objetivo foi de R\$ 8.916,47 com um custo de R \$ 5.999.80 em mercadorias. Para a obtenção do lucro nominal do comerciante, basta fazer a subtração do valor da função objetivo com o custo das mercadorias, obtendo-se assim um resultado de $\mathrm{R} \$ 2.916,68$. Vale lembrar que o comerciante tinha um fluxo de caixa de $\mathrm{R} \$$ 6.000,00 inicialmente. Em linhas gerais, o resultado foi condizente à realidade do negócio, tendo sido validado diretamente com o proprietário.

\section{REFERÊNCIAS}


AlMEIDA, S. O., BRASil, V. S., CAllegaro, A. R. C., CAllegarO, F. C. O contínuo experiencial do varejo: Valor utilitário versus valor hedôneo no mix de marketing $\quad$ varejista. $2016 . \quad$ Disponível em: http://meriva.pucrs.br/dspace/bitstream/10923/10320/2/O_Continuo_Experiencial_do_Varej o_Valor_Utilitario_Versus_Valor_Hedonico_no_Mix_de_Marketing_Varejista.pdf . Acesso em: 07/08/2018.

ARAUJO, C. L., MIRANDA, E. P., MOREIRA, A., CRUZ, F., SOUSA, J. S. O papel econômico, social, ambiental e cultural da feira do padre, aos olhos de seus frequentadores: O caso da feira do padre, em Sobradinho. Anais XIV Encontro da Luso-Brasileira de estudos ambientais. Recife/PE, 2011.

ARENAles, M., et al. Pesquisa Operacional para cursos de engenharia. 2.ed. Rio de Janeiro: Elsiever, 2015.

BELFIORE, P.; FÁVERO, L. P. Pesquisa Operacional para cursos de engenharia. Rio de Janeiro: Elsiever, 2013.

BRIESEMEISTER, M., BORBA, M. P. Programação matemática aplicada ao gerenciamento de projetos. Anais XXXIV Encontro Nacional de Engenharia de Produção. Curitiba/PR, 2014.

BRITO, G. D., NETO, E. G. L., REIS, B. T., RIBEIRO, V. S., BRANDAO, R. F. S. A influência do layout na comercialização em um mercado de médio porte. Anais XXXVII Encontro Nacional de Engenharia de Produção. Joinville/SC, 2017.

CHAMIE, B. C., IKEDA, A. A., PARENTE, J. Atmosfera de loja como um elemento da estratégia varejista. Revista Administração em Diálogo, Brasil, v. 14, n. 1, p. 01-31, 2012. CHAVES, G. R. Análise socioeconômica e cultural da feira livre do município de Remígio-PB.2011. 107 f. Monografia (Especialização) - Curso de Geografia, Departamento de Geografia, Universidade Estadual da Paraíba, Campina Grande-pb, 2011. Disponível em: $<$ http://dspace.bc.uepb.edu.br/jspui/handle/123456789/4044>. Acesso em: 21 mar. 2018.

CUNHA, J. G. A feira livre do município de Mari-PB: Uma análise histórica, geográfica e socioeconômica. 2014. 25 f. TCC (Graduação) - Curso de Geografia, Departamento de Geografia, Universidade Estadual da Paraíba, Paraíba, 2014. Disponível em: $<$ http://dspace.bc.uepb.edu.br/jspui/handle/123456789/4721>. Acesso em: 06 abr. 2018.

GUERRA, K. M., HEIZER, I. H., MOURA, A. S. D., MOURA, S. A., DELGADO, S. S. Gestão de estoque no setor de varejo de eletrodomésticos. Anais XX Simpósio de Engenharia de Produção. Bauru/SP, 2013. 
KOTLER, P. Administração de marketing. 12. Ed. São Paulo: Prentice Hall, 2006.

KOTLER, P; ARMSTRONG, G. Princípios de marketing. 12. ed. São Paulo: Pearson Prentice Hall, 2007.

LACHTERMACHER, G. Pesquisa Operacional na tomada de decisões. 3.ed. Rio de Janeiro: Elsevier, 2007.

MOREIRA, D. A. Pesquisa Operacional: curso introdutório. 2. Ed. São Paulo: Thomson Learning, 2013.

PEIXOTO, G. R. G., VASCONCElos, S. A., BARros, V. C. CONTAGEM, T. F., BACHEGA, S. J. Uso de programação linear inteira para maximização do lucro de uma distribuidora de bebidas. Anais XXXVII Encontro Nacional de Engenharia de Produção. SILVA, D. O., CASTRO, J. R. B., LOPES, K. P. S., SILVA, A. O. Caracterização e análise da feira livre de Cruz das Almas-BA sob a ótica do planejamento de gestão ambiental. Revista Caminhos de Geografia, Brasil, v.15, n.49, P. 01-13, 2014.

TEIXEIRA, N. B., SILVA, C. F., SOUZA, A., BACHEGA, S. J. Aplicação da programação linear inteira em uma pequena fábrica de churrasqueiras pré-moldadas. Anais XXIV Simpósio de Engenharia de Produção. Bauru/SP, 2017. 